\title{
CORRIGENDUM
}

\section{MiR-873 regulates ER $\alpha$ transcriptional activity and tamoxifen resistance via targeting CDK3 in breast cancer cells}

J Cui, Y Yang, H Li, Y Leng, K Qian, Q Huang, C Zhang, Z Lu, J Chen, T Sun, R Wu, Y Sun, H Song, X Wei, P Jing, X Yang and C Zhang

Oncogene (2015) 34, 4018; doi:10.1038/onc.2015.201

Correction to: Oncogene (2015) 34, 3895-3907; doi:10.1038/ onc.2014.430; published online 22 December 2014

Since the publication of the above article, the Department of Cancer and Cell Biology, University of Cincinnati, requested the authors who have listed the University in their affiliation to remove the affiliation from this publication as the study was not performed at the University and was not associated with their roles at the University.

As a result, the authors have requested Mingjun Bi, Anne-Marie Overstreet and Alex Meredith be removed from the author list and listed instead under the Acknowledgement section of the paper. Xingyuan Yang had already left the University of Cincinnati and carried out some work at his current institution, Institute of Health Sciences, Anhui University, thus the authors have requested his affiliation be updated.

The amended author list and acknowledgements section are shown below:

Authors: J Cui ${ }^{1}, \mathrm{Y}$ Yang ${ }^{2}, \mathrm{H} \mathrm{Li}{ }^{3}, \mathrm{Y}$ Leng $^{4}, \mathrm{~K}$ Qian ${ }^{4}, \mathrm{Q}$ Huang $^{5}$,

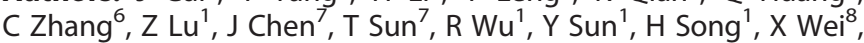

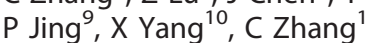

${ }^{1}$ Institute of Disease Control and Prevention, Chinese Academy of Military Medical Sciences, Beijing, China; ${ }^{2}$ Beijing Institute for
Neuroscience, Capital Medical University, Beijing, China; ${ }^{3}$ Department of Molecular \& Biomedical Pharmacology, University of Kentucky College of Medicine, Lexington, KY, USA; ${ }^{4}$ The Affiliated Hospital of Jiujiang University, Jiujiang, China; ${ }^{5}$ Department of Animal Sciences and Technology, Jilin Agriculture University, Changchun, China; ${ }^{6}$ Division of Experimental Hematology and Cancer Biology, Cincinnati Children's Hospital Medical Center, Cincinnati, OH, USA; ${ }^{7}$ Urology Department, the First Hospital of Nanchang University, Nanchang, China; ${ }^{8}$ Department of Applied Chemistry, College of Chemistry \& Molecular Engineering, Peking University, Beijing, China; ${ }^{9}$ Department of Chemistry, College of Arts and Sciences, Indiana University-Purdue University Fort Wayne Fort Wayne, IN, USA and ${ }^{10}$ Institute of Health Sciences, Anhui University, Hefei, China

\section{ACKNOWLEDGEMENTS}

We are grateful to Mingjun Bi, Anne-Marie Overstreet and Alex Meredith for their advice, discussion and editorial assistance. We thank Dr. Guanxuan Tan and his lab members for reagents and advice. We also thank Bill Evans for editorial support and for improving the English. This study was supported by the National Natural Science Foundation of China (Grant Nos. 81102005 and 31271154) and the National High Technology Research and Development Programme of China (Grant No. 2014AA020516). 